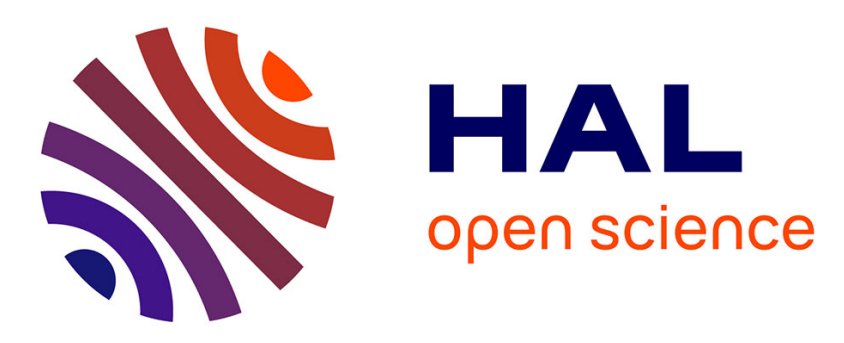

\title{
Vessel geometry modeling and segmentation using convolution surfaces and an implicit medial axis
}

Guillaume Pizaine, Elsa D. Angelini, Isabelle Bloch, Sherif Makram-Ebeid

\section{To cite this version:}

Guillaume Pizaine, Elsa D. Angelini, Isabelle Bloch, Sherif Makram-Ebeid. Vessel geometry modeling and segmentation using convolution surfaces and an implicit medial axis. 2011 IEEE International Symposium on Biomedical Imaging: From Nano to Macro (ISBI), Mar 2011, Chicago, Illinois, United States. pp.1421-1424, 10.1109/ISBI.2011.5872666 . hal-00614137

\section{HAL Id: hal-00614137 https://hal.science/hal-00614137}

Submitted on 9 Aug 2011

HAL is a multi-disciplinary open access archive for the deposit and dissemination of scientific research documents, whether they are published or not. The documents may come from teaching and research institutions in France or abroad, or from public or private research centers.
L'archive ouverte pluridisciplinaire HAL, est destinée au dépôt et à la diffusion de documents scientifiques de niveau recherche, publiés ou non, émanant des établissements d'enseignement et de recherche français ou étrangers, des laboratoires publics ou privés. 


\title{
VESSEL GEOMETRY MODELING AND SEGMENTATION USING CONVOLUTION SURFACES AND AN IMPLICIT MEDIAL AXIS
}

\author{
Guillaume Pizaine $^{1,2} \quad$ Elsa D. Angelini ${ }^{1} \quad$ Isabelle Bloch $^{1} \quad$ Sherif Makram-Ebeid $^{2}$ \\ ${ }^{1}$ Medisys Research Lab, Philips Healthcare, Suresnes, France. \\ ${ }^{2}$ Institut Telecom, Telecom ParisTech, CNRS LTCI, Paris, France.
}

\begin{abstract}
In the context of vessel tree structures segmentation with implicit deformable models, we propose to exploit convolution surfaces to introduce a novel variational formulation, robust to bifurcations, tangential vessels and aneurysms. Vessels are represented by an implicit function resulting from the convolution of the centerlines of the vessels, modeled as a second implicit function, with localized kernels of continuously-varying scales. The advantages of this coupled representation are twofold. First, it allows for a joint determination of the vessels centerlines and radii, with a single model relevant for segmentation and visualization tasks. Second, it allows us to define a new shape constraint on the implicit function representing the centerlines, to enforce the tubular shape of the segmented objects. The algorithm has been evaluated on the segmentation of the portal veins in 20 CT-scans of the liver from the 3D-IRCADb-01 database, achieving an average recovery of $73 \%$ of the trees with fast computational times.
\end{abstract}

Index Terms - vessel segmentation, convolution surface, arborescent structures, variational methods, shape constraint

\section{INTRODUCTION}

Fully automatic vascular tree segmentation is a challenging task that remains an active research field. Various dedicated segmentation formulations have been proposed: model-based (Krissian et al., 2000 [1]), explicit or implicit active contours (Lorigo et al., 1999 [2]) and stochastic tracking (Florin et al., 2005 [3]), for example (we refer to Lesage et al., 2009 [4] for an extensive survey on vessel modeling and segmentation). Segmentation algorithms usually extract the vessel boundary and recover the centerline of the vessels in two separate stages. Few research works, such as those by Deschamps et al., 2000 [5] or Li et al., 2009 [6], have attempted to address the problem of their joint extraction. As described in the seminal work by Bloomenthal et al., 1991 [7], convolution surfaces can be used as an alternative to model, manipulate and visualize these two geometric components.

Convolution surfaces are defined as the convolution of a shape primitive with a set of localized kernels and provide an implicit shape formulation similar to the envelope of spheres representation used by Li et al., 2009 [6]. In a recent work by Lefevre et al., 2010 [8], a single branch vessel model based on convolution surfaces was introduced. However, the explicit parameterization of the centerline hindered its extension to multi-branch vessel trees. In this paper, we propose to reformulate the segmentation problem with the use of an implicit representation of the centerline.

Lorigo et al., 1999 [2] extracted vessel structures via the localization and regularization of its centerlines $\Gamma$ only, which defined manifolds of co-dimension 2 in 3D. In practice, the vessel centerline was defined as the $\epsilon$-isolevel of a function $\Phi, \Gamma_{\epsilon}=\{\boldsymbol{x} \mid \Phi(\boldsymbol{x})=\epsilon\}$, where $\Phi$ was the signed distance function to a curve $\mathcal{C}$ and $\epsilon$ an arbitrarily small real positive number.

Using a similar concept, our first contribution consists in modeling the vessel centerlines as the isolevel of an implicit function. It provides us with a suitable representation that can evolve in a variational setting (Sect. 2.1). Sect. 2.2 details the initialization and the evolution of the convolution surface model. Our second contribution is a novel dedicated geometrical constraint designed to maintain the tubular shape of the segmented objects (Sect. 2.3). Preliminary results on 2D and 3D medical image data are presented and discussed in Sect. 3.

\section{VESSEL MODELING AND SEGMENTATION WITH AN IMPLICIT CENTERLINE}

\subsection{Shape modeling with convolution surfaces}

In Lefevre et al., 2010 [8], the authors introduced the use of convolution surfaces to model tubular structures evolving in a variational framework. Assuming circular cross-sections, the vessel shape was encoded with an open parameterized centerline $\boldsymbol{m}(s):[0,1] \rightarrow \mathbb{R}^{n}$ convolved with a set of pointwise localized kernels with continuously varying scales $\sigma(s)$. The corresponding two-parameters level set function was defined to encode the vessel contours:

$$
\Phi_{\boldsymbol{m}, \sigma}(\boldsymbol{x})=\int_{0}^{1} \omega\left(\frac{\|\boldsymbol{x}-\boldsymbol{m}(s)\|}{\sigma(s)}\right)\left\|\boldsymbol{m}^{\prime}(s)\right\| \mathrm{d} s-C,
$$

where $C$ is an arbitrary positive constant used to enforce negative values outside the vessels, and $\omega$ is a non-normalized Gaussian kernel $\omega(\boldsymbol{x})=\exp \left(-k \boldsymbol{x}^{2}\right)$, driven by the parameter $k \in \mathbb{R}^{+}$.

The vessel segmentation problem was then formulated as an optimal two-phase partition problem of the domain $\Omega$ of an image $I$, in which foreground (first phase) and background (second phase) correspond to the vessel tree and the surrounding structures, respectively. Relying on the intensity distributions $p_{i}, i=1,2$, of the foreground and the background, both regions were described by log-likelihood homogeneity measures $r_{i}(\boldsymbol{x})=-\log p_{i}(I(\boldsymbol{x}))$ previously used in Mory et al., 2007 [9]. The problem was solved by minimizing the following functional $E$ over the set of all possible image partitions $\{\mathcal{A}, \Omega \backslash \mathcal{A}\}$ :

$$
E=\mathcal{R}(\mathcal{A})+\int_{\mathcal{A}} r_{1}(\boldsymbol{x}) \mathrm{d} \boldsymbol{x}+\int_{\Omega \backslash \mathcal{A}} r_{2}(\boldsymbol{x}) \mathrm{d} \boldsymbol{x},
$$

where $\mathcal{R}(\mathcal{A})$ is a regularization term enforcing shape regularity.

In this work, we propose an alternative implicit formulation of the centerline to allow for natural evolutions of the vessels into arborescent shapes. The vessel boundaries are still modeled as the 


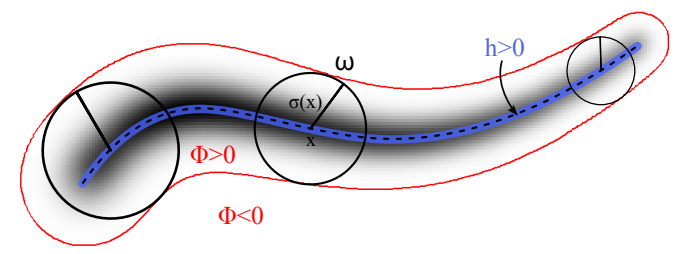

(a)

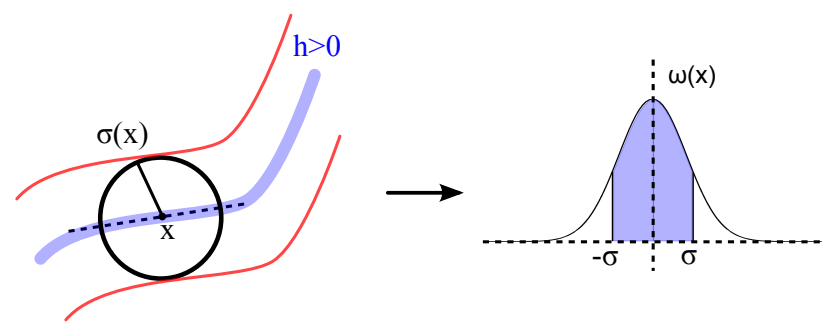

(b)

Fig. 1: Implicit representation of a tubular structure in 2D. (a) The foreground, within the red curve, is modeled as the convolution of a blue thin area $(h>0)$ localizing the vessel centerline, with local kernels $\omega$ of radius $\sigma$. (b) The area of the centerline lying inside a local kernel centered at $\boldsymbol{x}$ (on the left) is close to the blue-shaded area under the Gaussian profile (on the right).

zero-level set of a function $\Phi$ expressed as a convolution surface, but the centerline primitive is now the Heaviside function of a medialness function $h: \mathbb{R}^{n} \rightarrow \mathbb{R}$ (Fig. 1(a)):

$$
\Phi_{h, \sigma}(\boldsymbol{x})=\int_{\Omega} H(h(\boldsymbol{y})) \omega\left(\frac{\|\boldsymbol{x}-\boldsymbol{y}\|}{\sigma(\boldsymbol{y})}\right) \mathrm{d} \boldsymbol{y}-C .
$$

We chose to manipulate the Heaviside function of $h$ instead of the function itself to avoid the risk that many kernels may contribute to a single point on the vessel surface, which would alter the meaning of the optimized scale parameter $\sigma$. In practice, $C$ is set based on the assumption that on a straight cylinder, a point $\boldsymbol{x}_{\mathcal{S}}$ on the surface is generated by the contribution of only one kernel, and the distance to the center of this kernel is exactly $\sigma\left(\boldsymbol{x}_{\mathcal{S}}\right)$, hence $C=\omega(1)$.

\subsection{Extraction of medialness information with gradient diffu- sion}

For well-contrasted images, the initialization of the vessel segmentation typically consists of a single click inside the object and a corresponding estimation of the radius. However, on typical angiographic images, it proves crucial to provide a more accurate initialization for the initial region homogeneity measures. To this end, we resort to a Gradient Vector Flow (GVF)-based approach similar to the idea developed by Bauer et al., 2008 [10].

The GVF of an image is the vector field obtained by diffusing image gradients in uniform regions while keeping strong gradients untouched. The divergence of the resulting normalized vector field yields a map where high values characterize discontinuous orientations of the vector field, whereas small values identify regions with homogeneous directions. This enables to identify the centerlines of the structures as a subset of the ridges of this map. Centerlines are recovered by performing a height ridge traversal step, as described by Aylward et al., 2002 [11]. More precisely, seeds $\boldsymbol{s}_{\boldsymbol{i}}$ are selected among the local maxima of the map having image intensity values above the image median value. Using the second derivatives of the map, we compute an estimation of the local orientation $\boldsymbol{t}_{\boldsymbol{i}}^{\mathbf{0}}$ at seed points. Considering the neighbors $\boldsymbol{x}_{\boldsymbol{n}}$ of $\boldsymbol{s}_{\boldsymbol{i}}$ such that $\boldsymbol{t}_{\mathbf{0}} \cdot \boldsymbol{s}_{\boldsymbol{i}} \boldsymbol{x}_{\boldsymbol{n}}>0$, the point $\boldsymbol{x}_{\boldsymbol{n}}$ providing the highest value is selected as part of the centerline. This point selection is repeated until a point that has already been traversed is encountered, or until the medialness value decreases below a given threshold. Then, for each $\boldsymbol{c}_{\boldsymbol{i}}$ on a centerline, the local radius $\sigma\left(\boldsymbol{c}_{\boldsymbol{i}}\right)$ is estimated by following the GVF vector field from $c_{i}$ to the first local gradient extremum. A 2D example of the complete initialization data is illustrated in Fig. 2(b).

\subsection{Segmentation: energy functional and associated constraints}

Now that the model has been carefully initialized, the evolution is driven by the competition between the two homogeneity measures $r_{1}$ and $r_{2}$. Introducing our new implicit model into Eq.2, we reformulate the objective function as:

$$
E=\mathcal{R}(h, \sigma)+\int_{\Omega} H\left(\Phi_{h, \sigma}(\boldsymbol{x})\right) r(\boldsymbol{x}) \mathrm{d} \boldsymbol{x},
$$

where $r(\boldsymbol{x})=r_{1}(\boldsymbol{x})-r_{2}(\boldsymbol{x})$. Using standard calculus of variations and the generalized scaling property of the Dirac distribution, we derive the following gradient-descent scheme for $h$ and $\sigma$ :

$$
\begin{gathered}
\nabla_{h} E(\boldsymbol{y})=\delta(h(\boldsymbol{y})) \int_{\{\Phi=0\}} \tilde{r}(\boldsymbol{x}) \omega\left(\frac{\|\boldsymbol{x}-\boldsymbol{y}\|}{\sigma(\boldsymbol{y})}\right) \mathrm{d} \boldsymbol{x} \\
\nabla_{\sigma} E(\boldsymbol{y})=-\frac{H(h(\boldsymbol{y}))}{\sigma(\boldsymbol{y})^{2}} \int_{\{\Phi=0\}} \tilde{r}(\boldsymbol{x})\|\boldsymbol{x}-\boldsymbol{y}\| \omega^{\prime}\left(\frac{\|\boldsymbol{x}-\boldsymbol{y}\|}{\sigma(\boldsymbol{y})}\right) \mathrm{d} \boldsymbol{x},
\end{gathered}
$$

where $\tilde{r}=\frac{r}{\|\nabla \Phi\|}$.

In Eq.4, we need to define a regularization constraint on the spatial appearances of $h(\boldsymbol{x})$ and $\sigma(\boldsymbol{x})$. Since the geometry of the vessel is encoded with continuous spatial variables having smooth variations within the vessel and strong gradients at the vessel interface, we penalize the total variation (TV) norm of $h$ and $\sigma$ :

$$
\mathcal{R}(h, \sigma)=\lambda \int_{\Omega}\|\nabla h(\boldsymbol{x})\| \mathrm{d} \boldsymbol{x}+\mu \int_{\Omega}\|\nabla \sigma(\boldsymbol{x})\| \mathrm{d} \boldsymbol{x},
$$

where $\lambda, \mu \in \mathbb{R}^{+}$. Typically, we set $\lambda=0.1$ and $\mu=0.4$. High values of $\mu$ generate a smooth surface with slowly-varying radii, but the model may not be able to propagate into small branches, or to cope with partial stenosis. On the contrary, small values allow rapid variations of $\sigma$, but at the price of degrading the quality of the surface. Our initial experiments showed the need for additional constraints to prevent the medialness function $h$ from systematically spreading inside the object to be segmented.

Nain et al., 2004 [12] studied similar leakage problems of elongated implicit surfaces and introduced an effective volume constraint for a level set-based vessel segmentation framework. We are able to define an even more restrictive constraint by measuring the volume of $h$, instead of $\Phi$, inside a neighborhood of locally-adapted size defined by $\sigma$. Given a location $\boldsymbol{x}$ where $h(\boldsymbol{x})>0, h$ will be tubular around $\boldsymbol{x}$ if the corresponding local Gaussian kernel $\omega$ encompasses a volume of $h$ close to the volume of a straight tube going through its center (see Fig. 1(b)). This equivalent theoretical volume can therefore be expressed analytically as a function $V_{\sigma}$ of $\sigma(\boldsymbol{x})$ :

$$
V_{\sigma}(\boldsymbol{x})=\sigma(\boldsymbol{x}) \sqrt{\frac{\pi}{k}} \operatorname{erf}(\sqrt{k})
$$




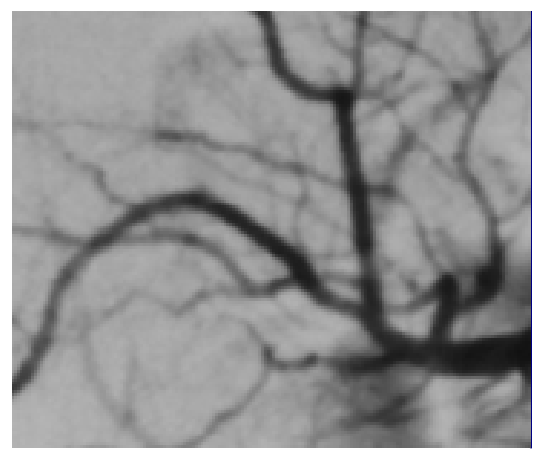

(a)

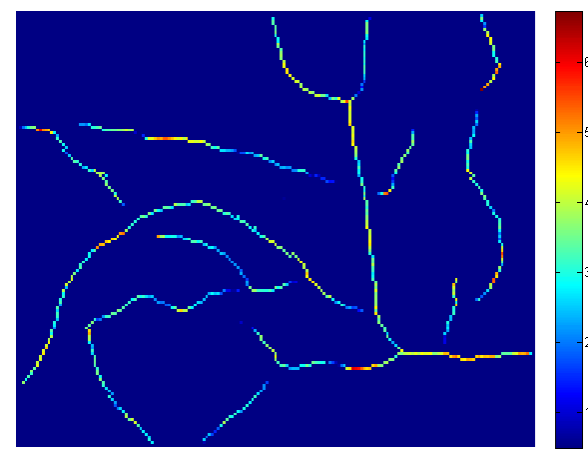

(b)

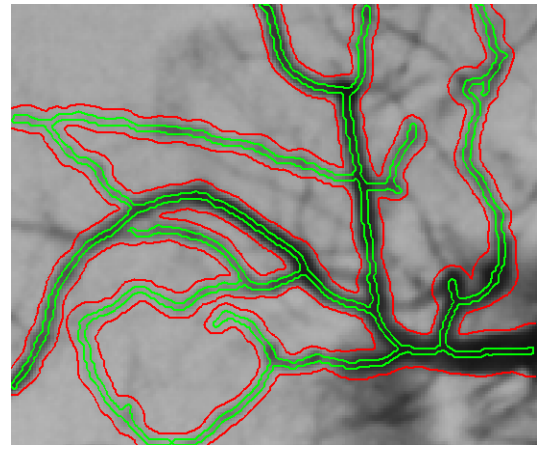

(c)

Fig. 2: Results on a 2D X-ray angiography with GVF-based initialization. (a) Original image. (b) Estimated centerlines and color-coded radii for initialization. (c) Final segmentation.

with erf is the error function and $k$ is the constant parameter of the Gaussian kernel (see Sect. 2.1). The effective volume of $h$ encompassed within $\omega$ is:

$$
V(\boldsymbol{x})=\int_{\Omega} H(h(\boldsymbol{y})) \omega\left(\frac{\|\boldsymbol{x}-\boldsymbol{y}\|}{\sigma(\boldsymbol{x})}\right) \mathrm{d} \boldsymbol{y} .
$$

Penalizing deviations from the expected value, we propose a novel volume constraint relying on the following elastic energy:

$$
E_{\mathrm{v}}=\frac{1}{2} \int_{\Omega}\left(V(\boldsymbol{x})-V_{\sigma}(\boldsymbol{x})\right)^{2} \mathrm{~d} \boldsymbol{x}
$$

Finally, we introduce a third term enforcing local alignment of the gradient of $h$ with the locally smoothed and normalized gradient of the image, at scale $\sigma: \boldsymbol{n}_{\sigma}=\nabla\left(I \star G_{\sigma}\right) /\left\|\nabla\left(I \star G_{\sigma}\right)\right\|$. This term is defined as:

$$
E_{m}=\frac{1}{2} \int_{\Omega}\left\|\nabla h(\boldsymbol{x})-\boldsymbol{n}_{\sigma}(\boldsymbol{x})\right\|^{2} \mathrm{~d} \boldsymbol{x} .
$$

Similarly to the gradient diffusion used during the initialization stage, this constraint quantifies the local asymmetry of the image gradients. Smoothing normalized gradients allows to identify basins where strong and aligned gradients prevail, and the boundaries between two such basins correspond to the locations of medial structures such as centerlines.

\section{PRELIMINARY RESULTS AND DISCUSSION}

In this section, we illustrate the performance of the proposed segmentation framework on $2 \mathrm{D}$ and $3 \mathrm{D}$ angiographic medical images (Fig. 2,3). An example of an initial set of centerlines and radii estimation for a 2D X-ray angiography is depicted in Fig. 2(b). The GVF-based initialization yields weak responses at bifurcations, especially when the intensities vary within the branches involved, which leads to disconnected centerlines. Fig. 2(c) illustrates the ability of our model to propagate into bifurcations and to reconnect branches of the vascular tree.

In Fig. 2(c), one can nevertheless notice how the tracking process misses a few bifurcations. This is due to the difficult tradeoff between regularity and accuracy on $\sigma$. On the one hand, allowing strong and rapid variations of the radii is paramount to recover small vessels branching off from much larger arteries. Regularity and shape constraints, on the other hand, are mandatory to maintain the consistency of the model, i.e. an equivalence between $\sigma$ and the real radius of the structures. Depending on the application, one may favor one or the other and set the weights of the different constraints accordingly.

Fig. 3 illustrates the above discussion with some preliminary $3 \mathrm{D}$ results on rotational angiographies (size $256 \times 256 \times 256$, spatial resolution $0.41 \times 0.41 \times 0.41 \mathrm{~mm}^{3}$ ) and 3D-CT scans of the liver (size $512 \times 512 \times 224$, spatial resolution $0.78 \times .078 \times 1.6 \mathrm{~mm}^{3}$ ) from the $3 \mathrm{D}$ IRCADb-01 database [13]. In the case of Fig. 3(a), strong constraints were applied to the model, resulting in a smooth segmentation and meaningful radii values. On the contrary, the segmentation in Fig. 3(b) was obtained using small weights for the volume constraint and the regularization over $\sigma$. Albeit more complete, the centerline of the recovered tree may spread into the whole vessels, especially into small branches (see for example the horizontal branch in the middle of Fig. 3(b) with a very ragged surface appearance due to many overlapping kernels). Fig. 3(c), 3(d) and 3(e) illustrate similar results for the segmentation of the portal veins in 3D-CT scans of the liver. Our first evaluation shows that $73 \%$ of the trees are recovered, on average. Small branches are often missed, due to the choice of parameters and the use of downsampled images.

Despite the challenging balance between the parameters of the model, the close-up pictures provided in Fig. 3(a) and 3(b) highlight two additional interesting features of the present work. Separating tangent vessels is often a difficult task since there is little or no contrast between the structures. Fig. 3 demonstrates that our model is robust to this setting and is able to generate distinct surfaces for tangent vessels. Moreover, our model can deal with aneurysms, which are finely segmented, with a smooth transition of $\sigma$ values.

\section{CONCLUSION}

We presented a novel region-based segmentation framework for treelike structures, based on a convolution surface representation, with two coupled implicit surfaces evolving in a level set setting. This representation is able to propagate naturally through bifurcations. The use of a continuous scale parameter allows us to estimate accurately the radii of the vessels. We showed that our approach performs well in challenging configurations such as tangent vessels and aneurysms. Ongoing work focuses on thorough quantitative evaluation of the segmentation results. Besides, we showed that, in the current model, the quality of the segmentation and of the underlying 


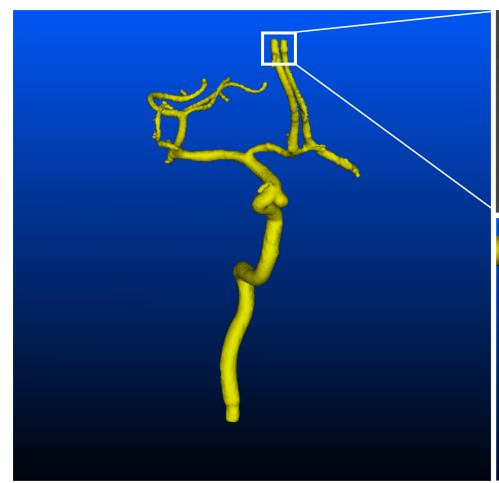

(a)

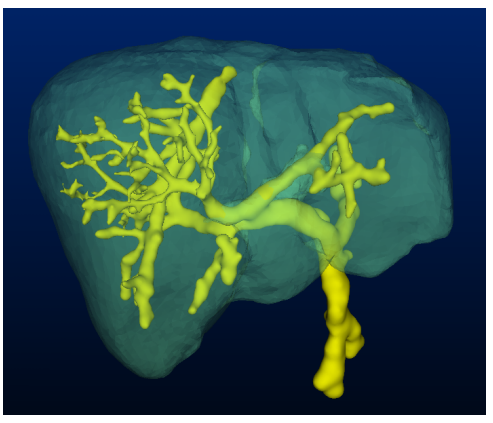

(c)
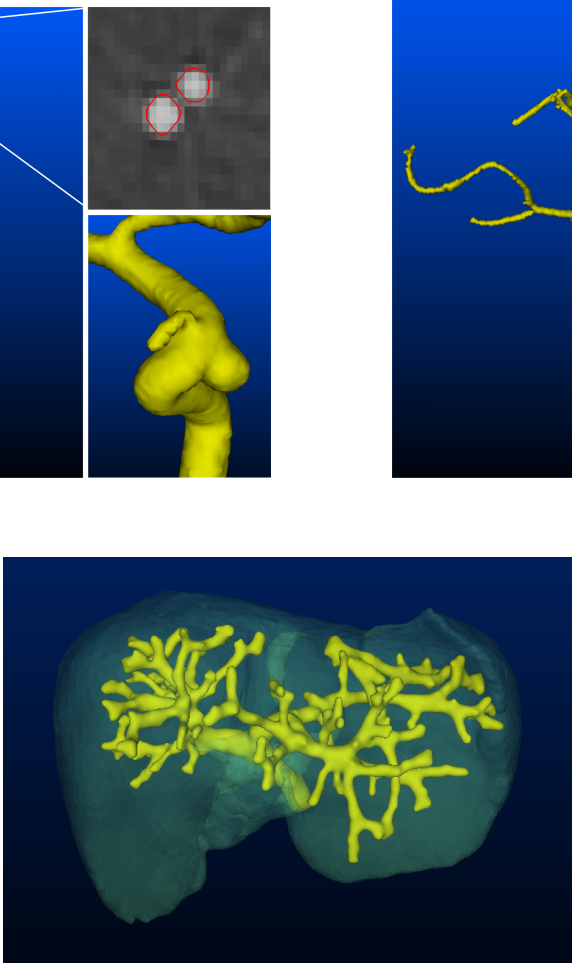

(d)
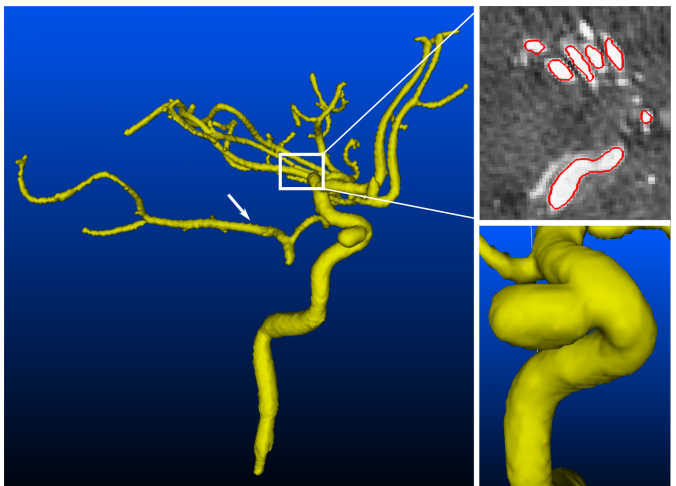

(b)

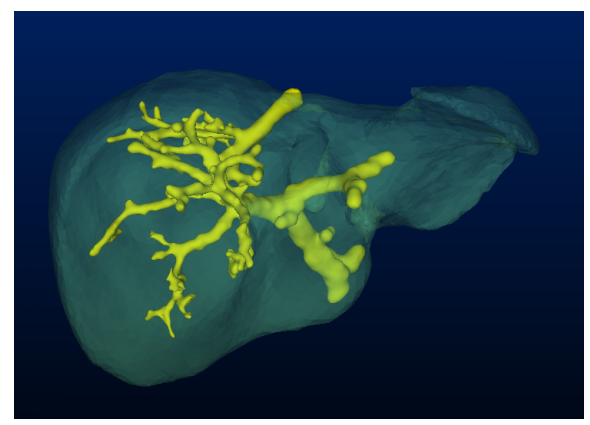

(e)

Fig. 3: (Top row) Results on two 3D rotational angiographies of the skull. For both segmentations of the arterial tree, we provide a 3D surface rendering view and two close-ups to demonstrate the ability of the algorithm to separate tangent vessels (top) and to cope with aneurysms (bottom). (Bottom row) Segmentation of the portal veins on 3D-CT scans of the liver (size 512x512x224, spatial resolution 0.78x.078x1.6 $\mathrm{mm}^{3}$ ). The segmentation is done on downsampled images, thus only branches larger than 2 voxels are recovered.

representation highly depends on the balance between the different regularization parameters. In the future, efforts should be put on developing a hierarchical approach to deal with structures presenting a wide range of scales, and to be robust to vessels presenting total occlusions.

\section{REFERENCES}

[1] Krissian, K., Malandain, G., Ayache, N., Vaillant, R. and Trousset, Y., "Model-based Detection of Tubular Structures," Computer Vision and Image Understanding, vol. 80, no. 2, pp. 130-171, 2000.

[2] Lorigo, L.M., Faugeras, O.D., Grimson, W.E.L., Keriven, R., Kikinis, R. and Westin, C.-F., "Co-dimension 2 Geodesic Active Contours for MRA Segmentation,” Proc. IPMI'99, pp. 126-139, 1999.

[3] Florin, C., Paragios, N. and Williams, J., "Particle Filters, a Quasi-Monte Carlo Solution for Segmentation of Coronaries," MICCAI'05, vol. LNCS 3749, pp. 246-253, 2005.

[4] Lesage, D., Angelini, E., Bloch, I. and Funka-Lea, G., "A Review of 3D Vessel Lumen Segmentation Techniques: Models, Features and Extraction Schemes," Medical Image Analysis, vol. 13, no. 6, pp. 819-845, 2009.

[5] Deschamps, T. and Cohen, L., "Minimal Paths in 3D Images and Application to Virtual Endoscopy," ECCV'00, vol. LNCS 1843, pp. 543-557, 2000.
[6] Li, H., Yezzi, A. and Cohen, L., "3D Multi-branch Tubular Surface and Centerline Extraction with 4D Iterative Key Points," MICCAI'09, vol. LNCS 5762, pp. 1042-1050, 2009.

[7] Bloomenthal, J. and Shoemake, K., "Convolution surfaces," Proc. SIGGRAPH'91, vol. 25, no. 4, pp. 251-256, 1991.

[8] Lefevre, T., Mory, B., Ardon, R., Sanchez-Castro, J. and Yezzi, A., "Automatic Inferior Vena Cava Segmentation in ContrastEnhanced CT Volumes,” Proc. ISBI'10, pp. 420-423, 2010.

[9] Mory, B., Ardon, R. and Thiran, J.-P., "Fuzzy Region Competition: A Convex Two-Phase Segmentation Framework," Proc. SSVM'07, vol. LNCS 4485, pp. 214-226, 2007.

[10] Bauer, C. and Bischof, H., "A Novel Approach for Detection of Tubular Objects and Its Application to Medical Image Analysis," 30th DAGM Symposium on Pattern Recognition (DAGM), 2008.

[11] Aylward, S.R. and Bullit, E., "Initialization, Noise, Singularities, and Scale in Height Ridge Traversal for Tubular Object Centerline Extraction," IEEE Transactions on Medical Imaging, vol. 21, no. 2, pp. 61-75, 2002.

[12] Nain, D., Yezzi, A. and Turk, G., "Vessel Segmentation Using a Shape Driven Flow," MICCAI'04, vol. LNCS 3216, pp. 5159, 2004.

[13] IRCAD, 3DIRCADb team, "3D-IRCADb-01 database," http: //www.ircad.fr/softwares/3Dircadb/ 3Dircadb1/index.php? lng=en. 